\title{
Artistic Conception and Object Selection in Jianjiang's Works
}

\section{Li Qiao}

Anhui University of Finance and Economics, Bengbu, Anhui, 230030

Keywords: Jian Jiang’s works, Artistic conception, Objects

\begin{abstract}
Jian Jiang's works are pieces of utmost beauty and sentiment which combines the characteristic of lofty mountains and rivers in paintings of the Song Dynasty, profound artistic conception in paintings of the Yuan Dynasty and unique writing style in paintings of the Ming and Qing Dynasty. His sentiment finds expression in the image forms, or cultural symbols in his paintings. The arrangement and design of those symbols are full of emotional connotations, rationed and irrational. Through the study of design and selection of image forms in his works, a more comprehensive understanding for him and his works can be obtained, it is hoped that the understanding of the selection and application of symbols in his works may provide reference for the analysis of other painters and their works.
\end{abstract}

Artistic conception and object selection should be consistent with the heart of the artist's presentation, and the artistic conception of classic works is created by most of the artist's elaborate design. Reading Jian Jiang's works can make one feels the clearness, quietness, and magnificence far away from the earth. Jian Jiang's works use various objects to create a spiritual paradise for himself and their audiences. Snowy mountains, empty houses, cold ponds and quiet pavilions are the frequently occurring objects in Jian Jiang's works. This paper will carry on the research and the analysis on the following two questions. First is the selection of these objects and their effects on the artistic conception of Jian Jiang's work. Second is whether are these objects chosen by Jian Jiang at hand or by the need to express his mood or the artistic conception of the works.

\section{Artistic Conception of Simplicity and Desolateness and Object Selection}

Dong Qichang said in his "Essay of the Room of Zen in Painting”: “Although there are many talents in the Yuan Dynasty, Yun Lin, style name of Ni Zan, is the one that features natural and unsophisticated in his works." Natural and unsophisticated is Dong Qichang's highest appraisal of Ni Zan. As a follower of Ni Zan, Jian Jiang endeavored to pursue the artistic charm of Ni Zan. However, due to the different background of the two, their aesthetic implication is also different. Jian Jiang's expression of the geometric shapes and his concise language all generate in people a sense of plain life, such as Chuziqingxiu, Qingmowusheng, Tuyijujing, all this give people a sense of eternity. If Ni Zan's landscapes reluctant to tell you all, then Jian Jiang's works are the embodiment of desire for nothing. Apart from showing the outline of the mountain through texture stroke, the rivers and mountains painted by Jian Jiang can hardly seen any texture strokes. The texture strokes used by many ancients are basically ignored in Jian Jiang's works. The works with less hooks and strokes showcase a morally lofty sense and the specific object symbols are set to imply the artistic conception of quietness and simplicity.

White snow for a Zen master means empty, morally lofty, and a world without dusts. Different artists have different moods, so does snow scenes. Mountains in the Ju Ran's painting "Snow” are clear and transparent, and the scene is warmly white. Huang Gongwang left the world with the painting "Jiufengxueji", "Kuaixueshiqing” and other works. Tasting these works is like entering into a fairyland, a color-glazed world with warm and clear snowy scene. The painter, Jian Jiang also painted snow. The shadow side of the sky and the rock were dyed in light black in most of his paintings about snow. Chine is integrated into light black to show the dark and gloomy sky. This type of technique of painting is adopted in his work named Huangshan Atlas of Wenshu Temple (See Figure 1). Brownish red is mixed with light black to express the hazy scenes in the work like 
Huangshan Atlas of Lion Grove. Both the hazy and gloomy scenes in Jian Jiang's painting of snow are lonely. The scene of snow is painted for himself rather than for other people. Mountain road unmanned and peak uninhabited houses are places where there are no human beings, where emotions like loneliness, pain, depression, longing and others can be accommodated. The hazy, gloomy and lonely of the painting symbolizes Jian Jiang's life experience. The personal and domestic feelings of joy or sadness can only be expressed and discharged through painting.

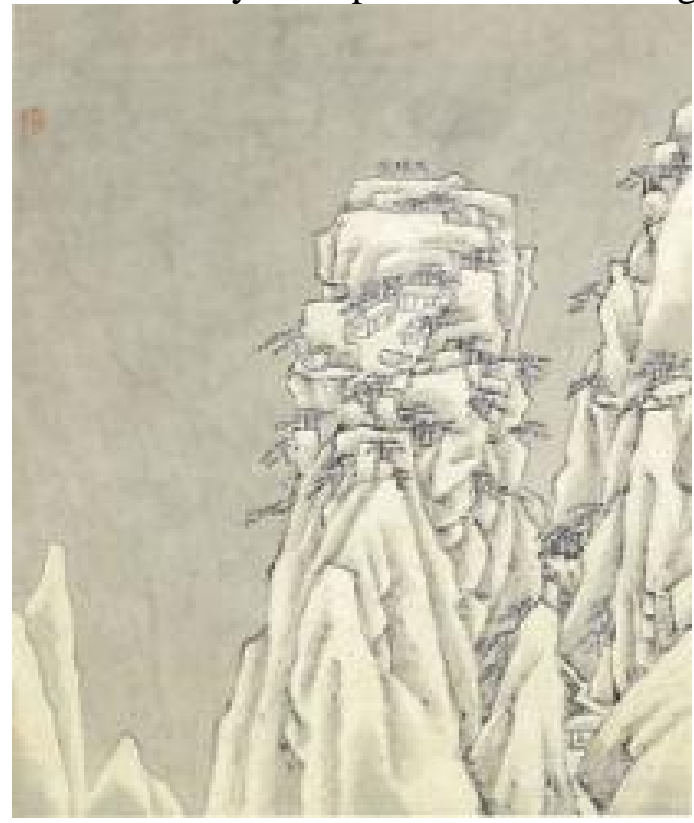

Figure 1

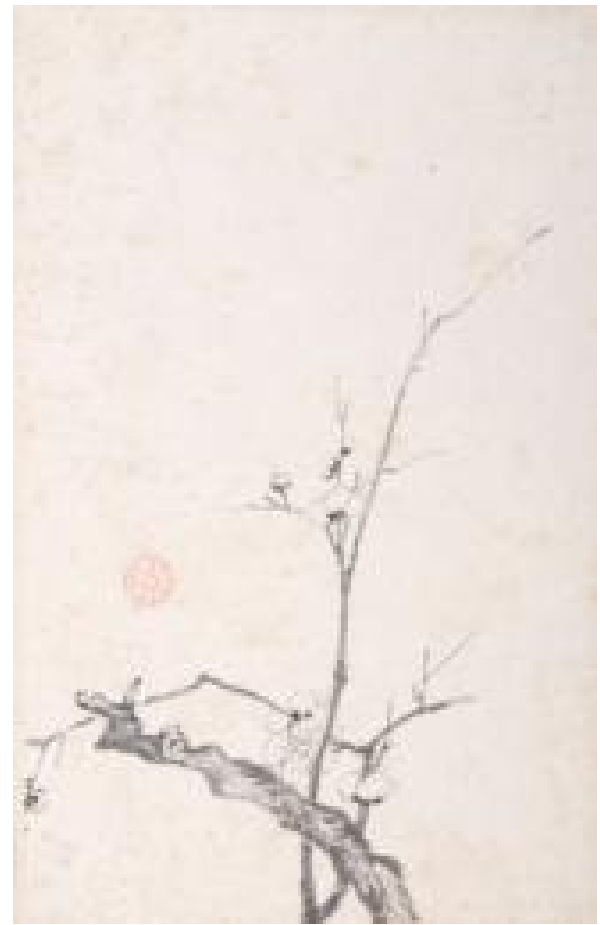

Figure 2

Plum blossom is also the image to express the feelings in the painting of Jian Jiang. Atlas of Songmei is collected in the Palace Museum in Beijing. Atlas of Mountains and Rivers and the Plum Blossom is enshrined in the Museum in Anhui province (See Figure 2). Jian Jiang loves plum blossom, paints it and writes for it. The shape of plum blossom in his painting is refined characterized by solitary branch and sparse shadow of flower. It is better to say that Jian Jiang chooses plum blossom, chooses the loneliness and elegance and magnificence of the withering 
greenery. The painter and the image correspond to each other in heart which refers to that the painting expresses emotional feelings. The description of sparse branch and flower reflects his escape to the miscellaneous world and his attachment to quiet and beauty. The bending branches and trunk and the exiguous plum blossom are able to consulate the painter's mind. The old and bending branches and trunk, growth ring and protrusion of stalk are the traces of sedimentation of years and invasion of flurry. The sparse flower embodies the soft feelings of the painter including cherishment, companion and consolation. The monk represents ruthlessness, being disillusioned with the mortal world and free from human desires and passions. While plum blossom affection symbolizes affection, being tough and tensile, pure and noble, overflowing with fragrance. Jian Jiang is the combination of the monk and plum blossom, the contradictory complex of affection and ruthlessness and resolution and cherishment. He cherishes freezing cold and quiet. Hence, only sparse plum blossom accompanys him day and night after his death.

Old raft and sliver grass are images often seen in Jian Jiang's work, he has a piece of work that is named "Picture of Old Raft and Sliver Grass". In the picture, there is an old man sitting alone in the cold house, in front of the house, two trees standing there in the courtyard one of which has lost all its leaves, the other seems dead, left only with the main branch. The wind whistling, the leaves sway, dead branches shock, squeak voice echoes, the people listening to the wind are meditating. For Jian Jiang, autumn is a season of loneliness and contemplation for life. There is also an uninhabited pool in the courtyard, a patch of silver grass are swaying in the wind which symbolizes the season autumn and the loneliness of the owner. The door of the house in the picture is closed and different from the half closed wooden door saving for the beloved ones which means hope, expectation and date, the full closed door symbolizes solitude, wait-free, desperation and loneliness. Sitting alone in the autumn, the unspoken heart of the author is shut just as the door. The courtyard in the cold autumn, the uninhabited pool, withered tree and sliver grass, the chosen of these lonely, sad images highlights the coldness of the autumn and the loneliness of the owner.

Empty house is a common image in Jian Jiang's works, it can be seen on the tops of the mountains in <Pine Tree and Snow in the West Rock>, <Wen Shu Temple of Yellow Mountain Drawing $>$, <Lion Forest $>$ and so on. As a supplement image, empty house can often be seen in the landscape painting; however, houses on the mountaintop can often lead to thinking, suspicion and uneasiness. Actually, the houses in the ancient paintings are usually located on the flat foot of the mountains, inside the houses, there are scholars reading, people playing the game of Go, or woman sitting beside the window. And yet the empty and quiet houses in Jian Jiang's works are on the top instead of on the foot of the mountains. Buildings on the mountaintop are mostly temples for the immortals. Houses are the homes for ordinary people. Temples belong to the monks and are open to the public while houses are private to their owners who are just ordinary people. Empty house means that the owner has already left, retired from the world or even dead. House is the symbolization of home, and yet home is just a dream for Jian Jiang, the empty house on the mountaintop is his home which he is not able to go back or rebuild. To Jian Jiang, poem and painting are the spiritual home to which his heart sticks, empty house on the mountaintop is his ideal resident, a place free from any worldly disturbance.

There is indifferent mood in Jian Jiang's works with aloof and solemn silence, attractive with free and quiet state rather than flirty beauty. The setting and selection of special images in his works complies with mental state and serves artistic conception of the paintings. Jian selects only a few desolate, quiet and indifferent images in harmony with inner emotion from the nature. His landscape paintings give a simple and appropriate summary.

\section{Grand and Profound Image Management}

Jian knows well about the brush-and-ink appeal of Southern School in terms of short scroll landscape paintings. While he grasps the modeling skills of Northern School and could paint multiple ranges of hills on the composition of the painting. Grand prospect and profound image management are expressed through relevant verbal signs. 


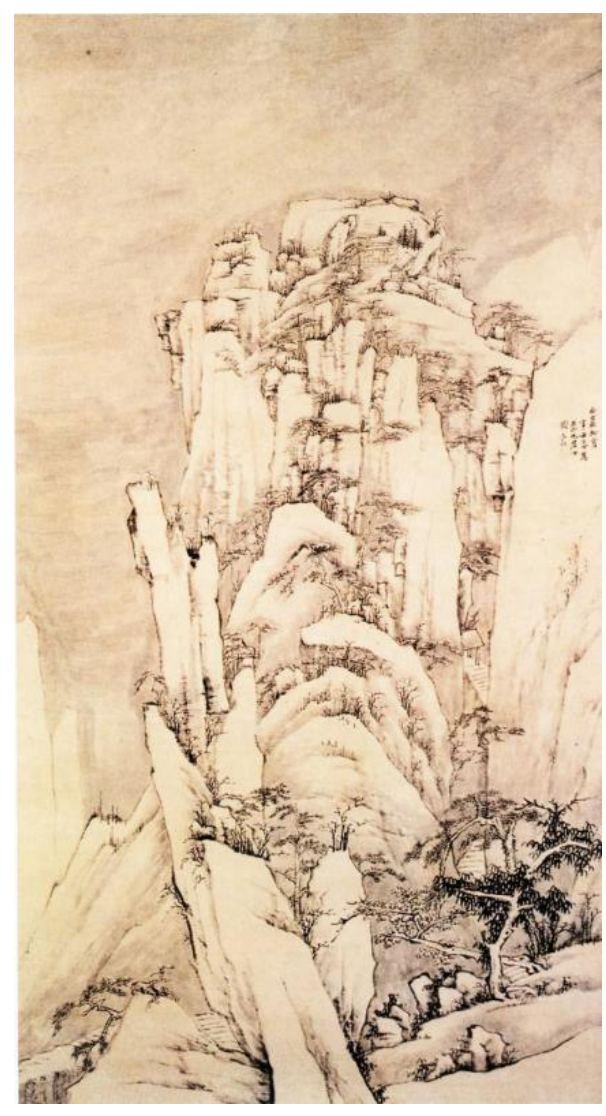

Figure 3

Jian's large-scale paintings include Tiandufeng Tu, Figure of pine and snow on West rock, Figure of pine and rock in Huanghai and Landscape given to Weimin. Figure of pine and snow on West rock (Figure 3) depicts the snowscape of Huangshan, whose composition could be divided into close-up, medium shot and long shot. In the close-up is a giant rock and lofty mountain, which tilts to the left of the picture, forming a thin, strange and steep Figure. The inclining inserted gesture disturbs other vertical peaks in the picture, causing a change between upright and slant in the composition. Peaks in the medium shot take up the most width of the screen, giving a rock-solid feeling. West rock in the long shot resembles cylinder and meets the clouds, whose magnificent shape makes the lofty mountain in close-up even more precipitous, constructing a strong visual contrast. On the right of West Rock, a cliff is painted using a line with brief texturing method, realizing the extreme simplicity, which combines with the mountain design and block portray of West Rock, causing a comparison of simplicity and complexity and making West Rock more prominent. A fitful mountain trail squeezes out between West Rock and the cliff. The hill gate is partly concealed, leading to a certain block relationship and a deep and serene picture. Various mountain shapes are covered and sheltered layer by layer, building a grand and profound picture image.

Depiction of giant rock and green pine can be found in Jian's works, whether large-scale masterpiece or short scroll with ample feelings. Some emphasize the hardness of rock against the sparse and pretty pines, while some depict coiled and entangled pine and rock. Rocks are immobile, ruthless, stubborn, and pines are coiled and distorted according to the shape of the rock. They are contending, recalcitrant, strangling, living and sentient. These works are like the image of the sculpture "Laocoon". All of them are repressive and protesting, twisting and crying. Only Laocoon's cry is tangible, as if he can hear the howl of pain, full of despair. However, the entanglement and struggle of Jian Jiang's rock pines are silent twists and turns, is unyielding, is lofty and tragic, full of tenacious vitality.

The people in Song Dynasty focused on the beauty of the object and especially the hills and valleys. Jian Jiang's works absorbed the hills and valleys' layouts of the people in Song Dynasty, made the composition of the layout in block, according to the landscape of Mount Huangshan. 
Jiang's picture comes from the nature, but it is used to express the feelings of the fortune in the chest. If the painting of Song Dynasty is closer to the delineation of nature and to make a true portrait, then Jiang's works write his heart history, pay more attention to the combination of passion and scenery, pay attention to the elucidation of the source of heart and the catharsis of emotion. The comprehension and application of Qiu He, who set his sights on the Song Dynasty, is an important aspect of the paintings feature of Wei Jun Chen Hou in Jiang's works.

\section{Conclusions}

Due to the understanding of the natural thing of Mount Huangshan, Jian's works all features magnificence and insipidness brought by mountains and gullies and withered branches. There are the beauty of nature, the beauty of mood and the beauty of drawing. Therefore, the works of Jian Jiang are the collection of Song, Yuan, Ming and Qing dynasties in one body into being perfect. Jian Jiang's works have the features both of stoic mood and magnificent picture composition. When operating the picture, Jian Jiang chose a specific object symbol, or humanistic symbols to show the arrangement and the design of these symbols is also emotional and rational, with rich emotional content, used to imply the emotional sustenance, to strengthen the mood of creation. Through the analysis of the object selection in his works, we can get a more comprehensive understanding of Jian Jiang, understand his works, read the artistic conceptions of his works, and provide some reference significance to the later painters' creation and appreciation.

\section{References}

[1] Wang Shiqing, Wang Cong: Jian Jiang Data Volume [M]. Hefei: Anhui People's Publishing, 1964, Page10.

[2] [Ming] Dong Qichang: Essay of the Room of Zen in Painting [M].

Shanghai: East China Normal University Press, 2012, Page79.

[3] Wang Shiqing, Wang Cong: Jian Jiang Data Volume [M]. Hefei: Anhui People's Publishing, 1964, Page40.

Author Introduction: (Anhui university of finance and economics school of the arts, Bengbu 23003) Mr. Riggio (1973). 